hep-th/0011226

CTP-MIT-3044

UUITP-11/00

\title{
Gauge Fields and Fermions in Tachyon Effective Field Theories
}

\author{
Joseph A. Minahan中 \\ Department of Theoretical Physics \\ Box 803, SE-751 08 Uppsala, Sweden \\ and \\ Barton Zwiebach \\ Center for Theoretical Physics \\ Massachusetts Institute of Technology \\ Cambridge, MA 02139, USA
}

\begin{abstract}
In this paper we incorporate gauge fields into the tachyon field theory models for unstable D-branes in bosonic and in Type II string theories. The chosen couplings yield massless gauge fields and an infinite set of equally spaced massive gauge fields on codimension one branes. A lack of a continuum spectrum is taken as evidence that the stable tachyon vacuum does not support conventional gauge excitations. For the bosonic string model we find two possible solvable couplings, one closely related to Born-Infeld forms and the other allowing a detailed comparison to the open string modes on bosonic D-branes. We also show how to include fermions in the type II model. They localize correctly on stable codimension one branes resulting in bose-fermi degeneracy at the massless level. Finally, we establish the solvability of a large class of models that include kinetic terms with more than two derivatives.
\end{abstract}

\footnotetext{
${ }^{1}$ E-mail: minahan@mit.edu

${ }^{2}$ E-mail: zwiebach@mitlns.mit.edu
} 


\section{Contents}

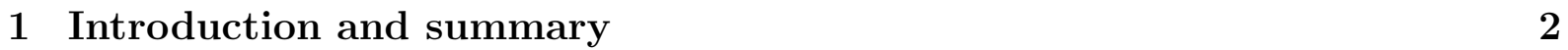

\begin{tabular}{|llll}
2 & Coupling gauge fields to the superstring tachyon model & 6
\end{tabular}

2.1 The superstring model revisited . . . . . . . . . . . . . . . . . . . . . . . 7

2.2 Coupling to gauge fields . . . . . . . . . . . . . . . . . . . . . . . . . . . 8

3 Coupling gauge fields to the bosonic string tachyon model 11

3.1 The bosonic string model revisited . . . . . . . . . . . . . . . . . . . . . 12

3.2 Coupling to gauge fields . . . . . . . . . . . . . . . . . . . . . . . 13

3.3 An alternative coupling to gauge fields . . . . . . . . . . . . . . . . . . . . 14

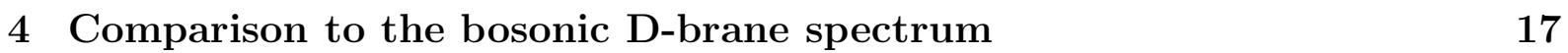

5 Fermion fields in the superstring tachyon model 20

6 Higher derivative actions in the superstring tachyon model 22

6.1 Higher derivative actions . . . . . . . . . . . . . . . . . . . . . . . 22

6.2 Gauge fluctuations for the modified Born-Infeld action . . . . . . . . . . . 25

6.3 Higher codimension branes $\ldots \ldots \ldots$. . . . . . . . . . . . . . . . 26

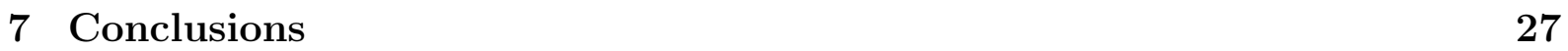

\section{Introduction and summary}

While the complete understanding of Sen's conjectures on tachyon condensation and Dbrane annihilation [1] is likely to require the full use of string field theory, simplified field theory models of tachyon dynamics are a useful tool in describing some aspects of this dynamics in a simpler context. In the familiar cubic version of open string field theory [2] (and its nonpolynomial extension to open superstring field theory [3]) the phenomenon of tachyon condensation is really a condensation of a string field; the tachyon, along with an infinite number of scalar modes acquire expectation values [4, 5, 6]. On the other hand, in boundary string field theory (B-SFT) [7, 8] - a much less developed version of string field theory incorporating a certain degree of background independence, tachyon condensation involves the tachyon field alone. Indeed, we proposed two models of tachyon dynamics [9, 10] that anticipated the exact tachyon potentials that emerge from 
analysis of B-SFT for both the open bosonic string and the open superstring respectively [11, 12, 13, 14]. Our models were derived by taking an $\ell \rightarrow \infty$ limit of tachyon models [15] whose spectrum around soliton solutions are governed by reflectionless Schroedinger potentials $U_{\ell}=\ell^{2}-\ell(\ell+1) \operatorname{sech}^{2}(x)$.

We included gauge fixed dynamics in the open bosonic string model of ref. [9]. These interactions, inspired by those of cubic string field theory considered in [18], give the expected results for the gauge field spectrum on the brane represented by a lump solution. Nevertheless, recent results in B-SFT including gauge fields [19, 20, 21, 22, 23] indicate that simpler gauge invariant interactions would also represent the physics quite accurately. One of the objectives of the present paper is to introduce into both the bosonic string and the superstring tachyon models gauge invariant interactions that preserve solvability and lead to localized gauge invariant dynamics on the world volume of solitons representing lower dimensional branes. Again, much of our insight is based on the behavior of finite $\ell$ models. Happily, our results are consistent with the general (Born-Infeld type) forms discussed in [19, 20, 21, 22, 23].

We will show that the gauge interactions in the superstring model lead to a discrete spectrum for the gauge fluctuations on the tachyon background representing the codimension one brane. In particular we find no continuum sector. The masses for the fluctuations match the masses found for the tachyon fluctuations. The coupling of the gauge fields is of the form $\exp \left(-T^{2} / 2\right) F_{\mu \nu}^{2}$. Since the tachyon vacuum is at $T= \pm \infty$ one may ask, as usual, whether the gauge field has dynamics on this vacuum. While the factor multiplying this gauge field is going to zero, suggesting that the gauge field becomes nondynamical and acts as a Lagrange multiplier that sets the associated currents to zero [24], this vanishing prefactor could alternatively be taken as a sign of strong coupling dynamics [25, 26] in which case the fate of the gauge field is less clear. We think, however, that the proof of gauge field localization on the codimension one brane, as evidenced by the discrete spectrum of gauge field fluctuations without a continuum, implies that the tachyonic vacuum does not support conventional local gauge field excitations. This is because the asymptotic regions of the codimension one brane live on the tachyon vacuum, and particle-like gauge fluctuations around this vacuum would have manifested themselves as a continuum spectrum of fluctuations of the gauge field around the lump solution.

For the bosonic case, we discuss two plausible gauge interaction terms. In the first one, arising from a Born-Infeld action, we find a discrete spectrum, where the spacing between

\footnotetext{
${ }^{3}$ For a pedagogical review on these and other solvable Hamiltonians with references to the early literature see 16]. Applications of reflectionless systems to fermions can be found in 17 .
} 
levels is twice the spacing for the tachyon fluctuations. For the second one the tachyon prefactor in front of the gauge kinetic term is the same as that for the tachyon kinetic term. Here we find that the level spacing matches those of the tachyon fluctuations. This second form of the coupling does not appear to have a solvable finite $\ell$ counterpart. Nevertheless, the correctness of the mass level spacing leads us to believe that this is the coupling chosen in string theory. The bosonic Born-Infeld action was considered in the recent papers of Cornalba [19], Okuyama [20], Andreev [21] and Tseytlin 23]. In particular, Andreev [21] discussed the possibility of ambiguities in such actions. The second form was actually advocated by Gerasimov and Shatashvili [22], as a natural choice of coordinates in the space of boundary interactions. While it seems likely that the two actions considered here would be related by a field redefinition in the context of an extended full theory including all higher derivatives, the truncations they represent do give different spectra.

Indeed, we use the spectrum associated to this second coupling to demonstrate how the fluctuation modes for the tachyon and gauge fields compare with open string states on bosonic D branes. In a complete string field theory model of tachyon condensation it is not enough to show that there is a discrete spectrum about the lump solutions. One must also account for the huge degeneracies that occur at the higher mass levels. In a theory with just a tachyon, there are no degenerate states at higher mass levels. If other fields are introduced, however, they too have fluctuations about the lump and modes can be degenerate. Moreover, an interesting and highly suggestive pattern emerges. The massless gauge field in the bulk localizes to a massless gauge field on the codimension one brane, with no extra massless scalar, as opposed to the case of Kaluza-Klein reduction, where a massless scalar accompanies the lower dimensional gauge field. String theory, however, still has this massless scalar, and in our framework it arises as a fluctuation mode of the tachyon field, the mode representing the translation of the brane. Such a pattern appears to repeat at higher mass levels and we believe it is generic: modes removed through localization reappear as fluctuation modes for lower mass fields. In light of these remarks, we now see that the equally spaced mass levels of the original tachyon models are more than just suggestive patterns. These patterns are necessary in order for the models to reproduce the full open string spectrum on a D-brane when additional fields are included. The result, with proper account of higher derivatives, is presumably B-SFT with all fields.

We also explore the coupling of fermions to the tachyon model of the superstring. Thus we include, along with the tachyon and the gauge field, the Majorana spinor present in the (unstable) D9 brane of IIA. We propose certain couplings of the tachyon field to the 
fermions that result in rather desirable properties. Namely, half of the fermionic degrees of freedom localize on the kink solution representing the D8, giving rise to a massless spectrum consistent with supersymmetry. The massive fermion spectrum is calculable and the mass squared spacing is the expected one. No continuum spectrum arises and the fermion becomes infinitely massive at the stable tachyonic vacuum.

Finally, we explore how higher derivative terms affect the solvability of the models. Focusing on the model relevant to the superstring we replace the derivative term $\partial T \partial T$ by a general function $f(\partial T \partial T)$ where $f^{\prime}(0) \neq 0$, and show that solvability is preserved while the precise values for the mass spacings and ratios of tensions between branes are altered. In particular, judicious choices of the form of $f$ allow us to obtain (solvable) Born-Infeld type actions. In particular we study a Born-Infeld type action proposed by Garousi [27] and Bergshoeff et.al. [28] where the tachyon kinetic term is insider the Born-Infeld square root. Such an action was argued to be consistent with open string scattering amplitudes and $T$ duality. We will find that this action has a kink solution with vanishing width. In addition, we find that the fluctuations for the tachyon field have the correct spacing between mass levels, although the spacings for gauge fluctuations is half the expected amount.

We will also see that higher derivative terms can lead to regular solutions for higher codimension branes. For example, with higher derivative terms there can be even codimension branes which are tachyon solitons of the world volume theory of several D9 brane-antibrane pairs of type IIB. Likewise, there can be odd codimension branes which are solitons of the world-volume theory of several unstable D9 branes of type IIA. In agreement with the observations of [13] such solutions do not appear to require expectation values for the gauge fields. The kinetic form of the tachyon field allows finite energy configurations even though the asymptotic values of the tachyon wind over the sphere at infinity.

We find it striking just how simple it is for the tachyon to induce complete localization for the gauge fields and fermions on the brane. From the viewpoint of solitons, we see that the complete localization of the tachyon relies on the absence of tachyon dynamics at the stable vacuum, something directly guaranteed by the form of the tachyon potential. This implies that the spectrum around the configuration representing the brane cannot have a continuum sector arising from the tachyon field. This results in a soliton spectrum of stringy type. Indeed, with the fluctuation spectrum governed by a Schroedinger potential with no continuous spectrum one must necessarily have an infinite number of energy levels. The complete localization of gauge fields and fermions follows by couplings to the 
localized tachyon. Again, these fields give rise to an infinite number of fields on the soliton. We find it quite remarkable that all this is exactly modeled with simple interactions of a scalar field, a gauge field and a fermion.

This paper is organized as follows. In section 2 we show how to include gauge fields through gauge invariant interactions for general classes of models with stable kinks, which include the two derivative truncation of the unstable D9 brane in type IIA. In section 3 we do a similar analysis for models with unstable lumps, which include the two derivative truncation for the tachyon effective field theory in bosonic string theory. In section 4 we compare the results for the bosonic gauge fluctuations to that of the bosonic string. Here, we are able to match the fluctuation modes to particular open string oscillator states. In section 5 we discuss the inclusion of fermion fields in the models with kinks. Finally in section 6 we consider the extension of the tachyon superstring model where we include certain kinds of higher derivative terms (this class includes the modified BornInfeld action). We then use such actions to obtain nontrivial solutions representing branes of codimension higher than one. Concluding remarks are offered in section 7 .

\section{Coupling gauge fields to the superstring tachyon model}

In Ref. [9], gauge fixed interactions were added to the bosonic tachyon effective field theory corresponding to the $\ell=\infty$ model, and to the finite $\ell$ models. Such interactions were inspired by similar terms appearing in gauge fixed cubic string field theory, and had been considered in [18]. The interactions were tailored to result in gauge fields localized on the tachyon solutions representing lower dimensional branes. The spectrum of massive gauge excitations could be solved exactly and were shown to have equally spaced levels, with the correct spacing. Nevertheless, it is now clear that in boundary string field theory one should naturally be led to a gauge invariant description of the interactions. Indeed, the forms of such interactions have recently been discussed by several authors [19, 20, 21].

In light of this, we look, for gauge invariant interactions of a gauge field with the tachyon field that lead to integer spacings for the gauge fluctuations, a residual gauge invariance on the solitons or lumps and absence of conventional degrees of freedom for the gauge field in the vacuum. There are remarkably simple actions that posess these features for both the bosonic model and the superstring model. In this section we will discuss the case of the superstring model while in section 3 will discuss the case of the the 
bosonic string model. In both cases it will be instructive to consider the finite $\ell$ models whose limit $\ell \rightarrow \infty$ led to the string models.

\subsection{The superstring model revisited}

Our construction will be quite general, allowing us to specialize to various models, such as the finite $\ell$ models discussed in [9], or to the harmonic oscillator model which is reached by taking the $\ell \rightarrow \infty$ limit and which leads directly to the two derivative truncation of boundary string field theory. As shown in [10], these models can be constructed in generality by reconstructing the field theory from the profile of the kink solution. Indeed, given a kink profile

$$
\bar{\phi}(x)=\mathcal{K}(x)
$$

where $\mathcal{K}(x)$, for kink $\mathrm{f}$, is either a monotonically increasing or a monotonically decreasing function of the coordinate $x \in[-\infty, \infty]$, the desired field theory is given by:

$$
S=-\int d t d^{p+1} x\left(\mathcal{K}^{\prime}(T)\right)^{2}\left((\partial T)^{2}+1\right)
$$

where the prime denotes partial derivative with respect to the argument, the scalar field $T$ is related to $\phi$ as

$$
\phi=\mathcal{K}(T)
$$

and the kink is just

$$
\bar{T}(x)=x .
$$

The action (2.2), assuming $\mathcal{K}^{\prime \prime}(0)=0$, has a vacuum at $T=0$ where there is a tachyon of mass squared

$$
M_{T}^{2}=\frac{\mathcal{K}^{\prime \prime \prime}(0)}{\mathcal{K}^{\prime}(0)}
$$

In addition, one easily confirms that

$$
\frac{\sqrt{2}}{2 \pi} \frac{\mathcal{T}_{\text {kink }}}{\mathcal{T}}=\frac{\sqrt{2}}{\pi} \int_{-\infty}^{\infty} d x\left(\frac{\mathcal{K}^{\prime}(x)}{\mathcal{K}^{\prime}(0)}\right)^{2},
$$

where $\mathcal{T}$ denotes the tension of the spacefilling brane represented by the $T=0$ vacuum of (2.2) and $\mathcal{T}_{\text {kink }}$ is the tension of the (codimension one) kink. In string theory the above ratio is one.

\footnotetext{
${ }^{4}$ In [10] we used the variable $\mathcal{P}$, for profile, rather than $\mathcal{K}$. We have changed notation to be able to call the lump profiles $\mathcal{L}(x)$.
} 
For example the finite $\ell$ models can be obtained by taking

$$
\mathcal{K}_{\ell}^{\prime}(x)=\sqrt{\mathcal{T}} \operatorname{sech}^{\ell}(x)
$$

The spectrum of fluctuations around the kink solutions of these models are governed by a Schroedinger equation with potential [9]

$$
U_{\ell}(x)=\ell^{2}-\ell(\ell+1) \operatorname{sech}^{2}(x) .
$$

We now consider the fluctuation problem around the kink solution $\bar{T}(x)=x$. For this purpose, we let $T \rightarrow x+\widetilde{T}$ in (2.2), where $\widetilde{T}$ represents the fluctuation field. It is then useful to redefine the fluctuation field as

$$
\mathcal{K}^{\prime}(x) \widetilde{T}=\widehat{T}
$$

Up to quadratic fluctuations and after dropping constant terms, the action is

$$
S_{\text {quad }}=-\int d t d^{p} y d x\left\{\partial_{\hat{\mu}} \widehat{T} \partial^{\hat{\mu}} \widehat{T}+\widehat{T}\left(-\frac{\partial^{2}}{\partial x^{2}}+\frac{\mathcal{K}^{\prime \prime \prime}(x)}{\mathcal{K}^{\prime}(x)}\right) \widehat{T}\right\} .
$$

The $p+2$ indices $\mu$ have been split into $p+1$ indices $\hat{\mu}$ along the brane worldvolume $\left(t, y^{i}\right)$, and the index $x$ along the coordinate transverse to the brane. From the above equation it follows that the Schroedinger problem for the tachyon fluctuations is simply

$$
-\frac{d^{2}}{d x^{2}} \widehat{T}+\frac{\mathcal{K}^{\prime \prime \prime}(x)}{\mathcal{K}^{\prime}(x)} \widehat{T}=M^{2} \widehat{T}
$$

The reader can verify that with $\mathcal{K}_{\ell}$ given in (2.7) the potential term in the above equation equals $U_{\ell}((2.8))$. It is also clear from the above equation that $\widehat{T}_{0}(x)=\mathcal{K}^{\prime}(x)$ is a solution with $M^{2}=0$. This is the translation mode of the kink in the original field variable. Indeed, from (2.3), (2.4) and (2.9) we have $\phi=\mathcal{K}\left(x+\epsilon \widehat{T}_{0}(x) / \mathcal{K}^{\prime}(x)\right)=\mathcal{K}(x)+\epsilon \mathcal{K}^{\prime}(x)+\mathcal{O}\left(\epsilon^{2}\right)$. Since $\mathcal{K}(x)$ is monotonic, $\mathcal{K}^{\prime}(x)$ does not have zeroes. Therefore, $\mathcal{K}^{\prime}(x)$ is the ground state wavefunction for the Schroedinger equation in (2.11) and thus there are no tachyonic fluctuations.

\subsection{Coupling to gauge fields}

In string theory, the gauge fields appear through the Born-Infeld action

$$
S_{B I}=-\mathcal{T} \int d t d^{p+1} x V(T) \sqrt{-\operatorname{det}\left(\eta_{\mu \nu}+F_{\mu \nu}\right)}
$$


where $V(T)$ is the tachyon potential. Here, we are only interested in finding the gauge fluctuations, so it suffices to make the approximation

$$
\sqrt{-\operatorname{det}\left(\eta_{\mu \nu}+F_{\mu \nu}\right)}=1+\frac{1}{4} F_{\mu \nu} F^{\mu \nu}+\ldots .
$$

Therefore, the action we use for the gauge fields and tachyons is

$$
S=-\int d t d^{p+1} x\left(\mathcal{K}^{\prime}(T)\right)^{2}\left((\partial T)^{2}+1+\frac{1}{4} F^{\mu \nu} F_{\mu \nu}\right) .
$$

In order to see that (2.14) is a reasonable coupling we study the spectrum of fluctuations of the gauge field on the background of the kink solution. We choose the axial gauge condition

$$
A_{x}=0, \quad \rightarrow F_{x \hat{\mu}}=\partial_{x} A_{\hat{\mu}} .
$$

We also note for later reference the equation of motion obtained by varying $A_{x}$, which in the chosen gauge simplifies to

$$
\partial_{\hat{\mu}}\left(\left(\mathcal{K}^{\prime}(T)\right)^{2} F^{x \hat{\mu}}\right)=0 \quad \rightarrow \quad \partial_{x}\left(\partial_{\hat{\mu}} A^{\hat{\mu}}\right)=0,
$$

where in the last step we have considered the reduction to linearized equations for gauge fluctuations on the background of the tachyon kink (the tachyon expectation value is independent of the brane coordinates). The term in the action (2.14) relevant to the gauge field fluctuations is

$$
S(\bar{T}, A)=-\int d t d^{p} y d x\left(\mathcal{K}^{\prime}(x)\right)^{2}\left(\frac{1}{4} F_{\hat{\mu} \hat{\nu}} F^{\hat{\mu} \hat{\nu}}+\frac{1}{2} \partial_{x} A_{\hat{\mu}} \partial_{x} A^{\hat{\mu}}\right) .
$$

The analysis is helped by the field redefinition

$$
B_{\hat{\mu}}=\mathcal{K}^{\prime}(x) A_{\hat{\mu}}, \quad \widetilde{F}_{\hat{\mu} \hat{\nu}}=\partial_{\hat{\mu}} B_{\hat{\nu}}-\partial_{\hat{\nu}} B_{\hat{\mu}},
$$

which allows us to turn (2.17) into

$$
S(\bar{T}, B(A))=-\int d t d^{p} y d x\left(\frac{1}{4} \widetilde{F}_{\hat{\mu} \hat{\nu}} \widetilde{F}^{\hat{\mu} \hat{\nu}}+\frac{1}{2} B_{\hat{\mu}}\left(-\frac{\partial^{2}}{\partial x^{2}}+\frac{\mathcal{K}^{\prime \prime \prime}(x)}{\mathcal{K}^{\prime}(x)}\right) B^{\hat{\mu}}\right) .
$$

Comparing this last equation with (2.10) we see that the Schroedinger problem governing the spectrum of the gauge field fluctuations on the kink is the same as that governing the tachyon fluctuations. Just as before the ground state is associated to the wavefunction $\mathcal{K}^{\prime}(x)$. Note that the constant in front in (2.19) plays no special role in this or the following analysis. 
It is straightforward to understand the gauge invariance of the massless gauge mode living on the lump solution. While we have imposed the axial gauge $A_{x}=0$, there is still a residual gauge invariance under

$$
A_{\hat{\mu}} \rightarrow A_{\hat{\mu}}+\partial_{\hat{\mu}} \varepsilon, \quad \text { with } \partial_{x} \varepsilon=0
$$

This means that the field $B_{\hat{\mu}}$ introduced in (2.18) transforms as

$$
B_{\hat{\mu}} \rightarrow B_{\hat{\mu}}+\mathcal{K}^{\prime}(x) \partial_{\hat{\mu}} \varepsilon
$$

The massless gauge field $B_{\hat{\mu}}(y)$ living on the brane arises as $B_{\hat{\mu}}(y, x)=B_{\hat{\mu}}(y) \mathcal{K}^{\prime}(x)$. It thus follows from the last equation that

$$
B_{\hat{\mu}}(y) \rightarrow B_{\hat{\mu}}(y)+\partial_{\hat{\mu}} \varepsilon, \quad \varepsilon=\varepsilon(y),
$$

which is the standard gauge transformation of a gauge field. Since for this massless mode $A_{\hat{\mu}}=B_{\hat{\mu}}(y)$ (see (2.18)) the subsidiary field equation in (2.16) is identically satisfied.

We can also confirm that the excited wave functions arising from the Schroedinger problem of (2.19) correspond to massive gauge fields on the brane. First note that the action for a mode

$$
B^{\hat{\mu}(n)}(y) \psi^{(n)}(x),
$$

where the wave function $\psi^{(n)}$ has eigenvalue $M_{n}^{2}$, is of the form

$$
\int d t d^{p} y\left(\frac{1}{4}\left(F_{\hat{\mu} \hat{\nu}}^{(n)}\right)^{2}+\frac{1}{2} M_{n}^{2}\left(B_{\hat{\mu}}^{(n)}\right)^{2}\right) .
$$

In addition, the constraint (2.16), using (2.18) and (2.23), reduces to

$$
\partial_{x}\left(\frac{\psi^{(n)}(x)}{\mathcal{K}^{\prime}(x)}\right) \partial_{\hat{\mu}} B^{\hat{\mu}(n)}(y)=0 \quad \rightarrow \quad \partial_{\hat{\mu}} B^{\hat{\mu}(n)}(y)=0 .
$$

The action (2.24), supplemented by this condition describes a massive gauge field.

Let us now turn to the example of the D9 brane. In [10, 13] it was argued that the lagrangian for the tachyon field for up to two derivatives is

$$
S=-\mathcal{T} \int d t d^{9} x \exp \left(-T^{2} / 2\right)\left[\partial_{\mu} T \partial^{\mu} T+1\right]
$$

This action can be obtained from (2.2) by setting

$$
\mathcal{K}^{\prime}(T)=\sqrt{\mathcal{T}} \exp \left(-T^{2} / 4\right)=\lim _{\ell \rightarrow \infty} \mathcal{K}_{\ell}(T / \sqrt{2 \ell})
$$


Since by construction, $\mathcal{K}^{\prime}(x)$ is a ground state wave function, we see that the corresponding potential is $U(x)=x^{2} / 4$. Given the potential term in (2.26), the Born-Infeld action is

$$
S_{B I}=-\mathcal{T} \int d t d^{9} x \exp \left(-T^{2} / 2\right) \sqrt{-\operatorname{det}\left(\eta_{\mu \nu}+F_{\mu \nu}\right)} .
$$

The integrand can be expanded as in (2.13) and so the fluctuation spectrum is found using the preceding arguments. Therefore, using (2.27) and (2.19), the action for the gauge fluctuations about the kink solution is

$$
-\mathcal{T} \int d t d^{8} y d x\left(\frac{1}{4} \widetilde{F}_{\mu \nu} \widetilde{F}^{\mu \nu}+\frac{1}{2} B_{\mu}\left(-\frac{\partial^{2}}{\partial x^{2}}+\frac{1}{4} x^{2}-\frac{1}{2}\right) B^{\mu}\right) .
$$

Hence, the fluctuation spectrum for the gauge fields is described by a harmonic oscillator, with the lowest mode corresponding to a massless state. The spacing between the modes is unity, which using (2.5) is twice the mass squared of the tachyon. As in (2.22) there is a residual gauge invariance for the massless vector mode on the stable 8-brane.

The absence of a continuum sector of gauge field fluctuations is manifest, and we take this as evidence that the tachyonic vacuum does not support conventional gauge field excitations. Indeed, in the case of scalar field excitations the logic connecting complete localization on the brane to lack of excitations on the stable vacuum is quite direct. The Schroedinger potential for fluctuations is $U(x)=V^{\prime \prime}(\bar{\phi}(x))=M_{e f f}^{2}(\bar{\phi}(x))$, where $V$ is the scalar field potential and $M_{e f f}^{2}$ denotes the effective mass of the scalar field (computed by cancelling the linear term, if nonvanishing, with an external source). We then have that $U(x \rightarrow \pm \infty)=M_{T}^{2}$ where $M_{T}^{2}$ denotes the scalar field mass at the stable vacuum. Thus a continuum arises in the Schroedinger problem for energies greater than $M_{T}^{2}$. If $M_{T}^{2} \rightarrow \infty$ there is no continuum.

\section{Coupling gauge fields to the bosonic string tachyon model}

In this section we discuss the general construction for gauge fields coupled to the bosonic string model. We will give two types of interaction terms. The first such term leads to a solvable spectrum for all finite $\ell$ models. This type of interaction is derivable from the Born-Infeld action. The second such term only has a solvable spectrum for the $\ell=\infty$ model. However, its spectrum is what one would expect from open string modes on D branes in bosonic string theory. This leads us to believe that this is the choice taken by B-SFT. 


\subsection{The bosonic string model revisited}

We begin by describing a general construction of a scalar field theory given the profile of a lump solution. We let

$$
\bar{\phi}(x)=\mathcal{L}\left(x^{2}\right)
$$

define the lump profile. By lump, we mean a field configuration where the derivative of the profile with respect to the coordinate $x$ vanishes at one point. The argument of $\mathcal{L}$ manifestly incorporates an expected reflection symmetry. The equation of motion of the lump relates the potential $V$ in the action to the spatial derivative of the profile as follows

$$
V(\bar{\phi}(x))=\frac{1}{2}\left[\bar{\phi}^{\prime}(x)\right]^{2}=2 x^{2}\left[\mathcal{L}^{\prime}\left(x^{2}\right)\right]^{2} .
$$

From (3.2) it is clear that $x \mathcal{L}^{\prime}\left(x^{2}\right)$ must have one and only one zero.

We now introduce a new field $\phi=\mathcal{L}(T)$, which in view of (3.1) implies

$$
\bar{T}(x)=x^{2} .
$$

This in turn gives $V(\bar{\phi})=2 \bar{T}\left[\mathcal{L}^{\prime}(\bar{T})\right]^{2}$. Thus, the action $S=-\int\left(\frac{1}{2}(\partial \phi)^{2}+V(\phi)\right)$ becomes

$$
S=-\int d t d^{p+1} x\left(\mathcal{L}^{\prime}(T)\right)^{2}\left(\frac{1}{2}(\partial T)^{2}+2 T\right) .
$$

For the finite $\ell$ models, we have that

$$
\mathcal{L}_{\ell}\left(x^{2}\right)=\sqrt{\frac{\mathcal{T}}{2 T_{0}}} \frac{1}{\operatorname{sech}^{\ell-1}\left(\sqrt{T_{0}}\right)} \operatorname{sech}^{\ell-1}(x),
$$

where $T_{0}$ is the value for $T$ at the unstable vacuum, where the potential is a maximum. The tension $\mathcal{T}$ is its vacuum energy.

To find the fluctuations, we let $T=x^{2}+\tau$ and define the fluctuation field as $\widehat{T}=$ $\mathcal{L}^{\prime}\left(x^{2}\right) \tau$. Up to quadratic order, the action in (3.4) becomes

$$
S=-\int d t d^{p+1} x\left(\frac{1}{2} \partial_{\hat{\mu}} \widehat{T} \partial^{\hat{\mu}} \widehat{T}+\frac{1}{2} \widehat{T}\left(-\frac{\partial^{2}}{\partial x^{2}}+\mathcal{U}(x)\right) \widehat{T}\right)
$$

where

$$
\mathcal{U}(x) \equiv 6 \frac{\mathcal{L}^{\prime \prime}\left(x^{2}\right)}{\mathcal{L}^{\prime}\left(x^{2}\right)}+4 x^{2} \frac{\mathcal{L}^{\prime \prime \prime}\left(x^{2}\right)}{\mathcal{L}^{\prime}\left(x^{2}\right)}=\frac{1}{x \mathcal{L}^{\prime}\left(x^{2}\right)} \frac{d^{2}}{d x^{2}}\left(x \mathcal{L}^{\prime}\left(x^{2}\right)\right)
$$

Hence, we see that the fluctuation spectrum is determined by the Schroedinger equation

$$
-\frac{d^{2}}{d x^{2}} \widehat{T}+\mathcal{U}(x) \widehat{T}=M^{2} \widehat{T} .
$$


It follows from the last equation in (3.7) that $x \mathcal{L}^{\prime}\left(x^{2}\right)$ is an eigenfunction with $M^{2}=0$, and so is the translation mode. Since $x \mathcal{L}^{\prime}\left(x^{2}\right)$ has one zero, this is the first excited state and therefore there exists one tachyonic mode.

We can now apply this general analysis to the specific case of the two derivative truncation of boundary string field theory. In boundary string field theory it was argued that the action for up to two derivatives for the tachyon field is [11, 12]

$$
S=-4 e \mathcal{T} \int d t d^{p+1} x\left\{\frac{1}{2} \partial_{\mu} \phi \partial^{\mu} \phi-\frac{1}{4} \phi^{2} \ln \phi^{2}\right\}
$$

This model can be obtained by taking the limit $\ell \rightarrow \infty$ in the finite $\ell$ models [9]. The field redefinition $\phi=\exp \left[-\frac{1}{4} T\right]$ casts this action in the form:

$$
S_{T}=-\frac{e \mathcal{T}}{4} \int d t d^{p+1} x \exp (-T / 2)\left[\frac{1}{2} \partial_{\mu} T \partial^{\mu} T+2 T\right] .
$$

This action has the form in (3.4), with

$$
\mathcal{L}^{\prime}(T)=\frac{1}{2} \sqrt{\mathcal{T} e} \exp (-T / 4)
$$

and hence has a codimension lump solution with $T=x^{2}$. From (3.11) and (3.7) we see that

$$
\mathcal{U}_{\infty}(x)=\frac{1}{4} x^{2}-\frac{3}{2}
$$

hence, the fluctuation spectrum has a tachyon with $m^{2}=-1$ and higher mass states with integer spacing, $\Delta m^{2}=1$. In [9] it was shown that the action in (3.10) also has codimension $d$ lump solutions with $T=\sum_{i}^{d}\left(x_{i}^{2} / 2\right)-2(d-1)$. In this case, the fluctuation spectrum is governed by a $d$ dimensional harmonic oscillator.

\subsection{Coupling to gauge fields}

Now let us include contributions from the gauge fields. We are again interested in the Born-Infeld action in (2.12), but for the analysis of the fluctuation spectrum it is sufficient to use

$$
S=-\int d t d^{p+1} x\left(\mathcal{L}^{\prime}(T)\right)^{2}\left(\frac{1}{2}(\partial T)^{2}+2 T+2 T \cdot \frac{1}{4} F^{\mu \nu} F_{\mu \nu}\right) .
$$

To find the fluctuation spectrum about the lump solution, we choose the axial gauge $A_{x}=0$ and let

$$
B_{\hat{\mu}}=(2 T)^{1 / 2} \mathcal{L}^{\prime}(T) A_{\hat{\mu}}, \quad \quad \widetilde{F}_{\hat{\mu} \hat{\nu}}=\partial_{\hat{\mu}} B_{\hat{\nu}}-\partial_{\hat{\nu}} B_{\hat{\mu}} .
$$

\footnotetext{
${ }^{5}$ We choose to work with the form of the 2 -derivative tachyon action for which the tachyon mass is the correct one.
} 
In terms of these fields, the part of the action containing the gauge fields becomes

$$
S(\bar{T}, B(A))=-\int d t d^{p} y d x\left(\frac{1}{4} \widetilde{F}^{\hat{\mu} \hat{\nu}} \widetilde{F}_{\hat{\mu} \hat{\nu}}+\frac{1}{2} B_{\hat{\mu}}\left(-\frac{\partial^{2}}{\partial x^{2}}+\mathcal{U}(x)\right) B^{\hat{\mu}}\right),
$$

where $\mathcal{U}(x)$ is defined in (3.7). Hence, the fluctuation spectrum for the $B_{\hat{\mu}}$ fields is governed by the same Schroedinger equation as for the tachyon fluctuations.

Since the Schroedinger potentials are the same, it would seem that the lowest mode for the gauge field is tachyonic. But this solution needs to be discarded, along with all solutions that are even under $x \rightarrow-x$. Upon inspection of (3.14), we see that $A_{\mu} \sim 1 / x$ if $B_{\mu}$ is nonzero at $x=0$, which is the case for the even parity modes. Therefore, the integrand in (3.13) diverges as $1 / x^{2}$, and so the even parity modes should be disregarded. Hence, the lowest mode corresponds to the first excited state of the Schroedinger equation and thus is massless.

Turning to the specific example of the two derivative truncation of B-SFT, the BornInfeld action has the form

$$
-\frac{e \mathcal{T}}{4} \int d t d^{p+1} x 2 T \exp (-T / 2) \sqrt{-\operatorname{det}\left(\eta_{\mu \nu}+F_{\mu \nu}\right)}
$$

This form of the action has been recently obtained in BSFT [19, 20, 21]. The overall normalization in (2.12) is chosen so that the gauge kinetic term has the canonical coefficient at the open string vacuum $T=2$, and so matches the form in (3.13) with $\mathcal{L}^{\prime}(T)$ defined in (3.11). Therefore, the term in (2.12) expanded to two derivatives can be written as

$$
-\int d t d^{p} y d x e^{-\bar{T} / 2}\left(\frac{1}{4} \widetilde{F}_{\hat{\mu} \hat{\nu}} \widetilde{F}^{\hat{\mu} \hat{\nu}}+\frac{1}{2} B_{\hat{\mu}}\left(-\frac{\partial^{2}}{\partial x^{2}}+\frac{1}{4} x^{2}-\frac{3}{2}\right) B^{\hat{\mu}}\right)
$$

where $\bar{T}$ refers to the tachyon field on the lump. Hence, the components of the gauge field also have a fluctuation spectrum given by a harmonic oscillator.

As before, the even parity solutions need to be discarded since they correspond to singular configurations for the gauge field $A_{\hat{\mu}}$. Hence the lowest mode is massless and the spacing between mass squared levels is equal to twice the magnitude of the mass squared term of the original tachyon.

\subsection{An alternative coupling to gauge fields}

The double spacing for the levels seen at the end of the last subsection seems odd and is perhaps a consequence of our only considering contributions of up to two derivatives. 
Therefore, let us also consider the gauge coupling

$$
S^{\prime}=-\int d t d^{p+1} x\left(\mathcal{L}^{\prime}(T)\right)^{2} \frac{1}{4} F_{\mu \nu} F^{\mu \nu} .
$$

This does not quite have the Born-Infeld form since it is missing a factor of $2 T$. Note that this is the simplest kind of term, the same factor multiplying the tachyon kinetic term is now multiplying the conventional gauge kinetic term.

To find the spectrum about the codimension one brane, we set $T=x^{2}$ and define the new gauge field $B_{\hat{\mu}}$ and associated field strength to be

$$
B_{\hat{\mu}}=\mathcal{L}^{\prime}\left(x^{2}\right) A_{\hat{\mu}}, \quad \widetilde{F}_{\hat{\mu} \hat{\nu}}=\partial_{\hat{\mu}} B_{\hat{\nu}}-\partial_{\hat{\nu}} B_{\hat{\mu}} .
$$

Subtituting back into (3.18) we find

$$
-\int d t d^{p} y d x\left(\frac{1}{4} \widetilde{F}_{\hat{\mu} \hat{\nu}} \widetilde{F}^{\hat{\mu} \hat{\nu}}+\frac{1}{2} \partial_{x} B_{\hat{\mu}} \partial_{x} B^{\hat{\mu}}+\frac{1}{2} U(x) B_{\hat{\mu}} B^{\hat{\mu}}\right),
$$

where $U(x)$ is given by

$$
U(x)=2 \frac{\mathcal{L}^{\prime \prime}\left(x^{2}\right)}{\mathcal{L}^{\prime}\left(x^{2}\right)}+4 x^{2} \frac{\mathcal{L}^{\prime \prime \prime}\left(x^{2}\right)}{\mathcal{L}^{\prime}\left(x^{2}\right)}=\frac{1}{\mathcal{L}^{\prime}\left(x^{2}\right)} \frac{d^{2}}{d x^{2}} \mathcal{L}^{\prime}\left(x^{2}\right) .
$$

This is a different Schroedinger potential than in (3.7) and does not seem to give a solvable spectrum for the gauge fields in the finite $\ell$ models.

For the $\ell=\infty$ model, however, the coupling in (3.18) still gives a solvable spectrum for the gauge fluctuations. Using the expression for $\mathcal{L}^{\prime}(T)$ in (3.11), the gauge action is

$$
S^{\prime}=-\frac{e \mathcal{T}}{16} \int d t d^{p+1} x \exp (-T / 2) F_{\mu \nu} F^{\mu \nu} .
$$

This form of the action has been recently advocated in [22]. Using (3.21), we have that

$$
U(x)=\frac{1}{4} x^{2}-\frac{1}{2} .
$$

Therefore, in contrast to the previous case, we find that the lowest mode for this harmonic oscillator equation is massless. In fact, there is no need to throw out the even parity modes, since these do not correspond to singular configurations for $A_{\hat{\mu}}$. Thus, the spacing between the gauge modes is the mass squared of the tachyon.

As in the superstring case, selecting a gauge imposes a constraint on the remaining fields. Proceeding as in (2.25) one finds the constraint on the massive modes

$$
\partial_{x} \partial^{\hat{\mu}}\left(\exp (\widetilde{T} / 2) B_{\hat{\mu}}^{(n)}\right)=0,
$$


where $\widetilde{T}$ refers to the tachyon field for the effective theory on the lump. For the effective field theory on the $p$ brane, this constraint on the modes can be implemented through a Stueckelberg action, familiar from gauge invariant free string field theory [29],

$$
-\int d t d^{p} y \exp (-\widetilde{T} / 2)\left(\frac{1}{4} \widetilde{F}_{\hat{\mu} \hat{\nu}}^{(n)} \widetilde{F}_{\hat{\mu} \hat{\nu}}^{(n)}+\frac{1}{2} n\left(B_{\hat{\mu}}^{(n)}-\partial_{\hat{\mu}} \phi^{(n)}\right)^{2}\right) .
$$

Using the action in (3.25) we can now study the reduction of a massive gauge field on the $p$-brane down to a $(p-1)$-brane. We will show that each massive gauge field on the $p$-brane gives rise to an equally massive gauge field in the $(p-1)$-brane, and at equally spaced higher mass levels, a massive gauge field plus a massive scalar.

To this end, we consider the $p-1$ brane solution $\widetilde{T}=w^{2}-2$ of the $p$ brane field theory. The massless mode $B_{\hat{\mu}}^{(0)}$ on the $p$-brane works exactly like the massless gauge field on the $(p+1)$-brane. Hence the fluctuation spectrum for this mode has a massless gauge field on the $(p-1)$-brane and equally spaced massive vector fields above this. The lost massless component, corresponding to the transverse gauge component, is compensated for by the massless tachyon mode, so that the total number of massless degrees of freedom remains the same.

Next consider the massive vector fields on the $(p-1)$-brane. We let barred indices $\bar{\mu}, \bar{\nu}$, run over the $p$ world volume coordinates of the $(p-1)$-brane. Let us impose the axial gauge $B_{w}^{(n)}=0$ and define new fields $C_{\bar{\mu}}^{(n)}=\exp \left(-w^{2} / 4\right) B_{\bar{\mu}}^{(n)}, \widetilde{G}_{\bar{\mu} \bar{\nu}}=\partial_{\bar{\mu}} C_{\bar{\nu}}-\partial_{\bar{\nu}} C_{\bar{\mu}}$ and $\varphi^{(n)}=\exp \left(-w^{2} / 4\right) \phi^{(n)}$. Then the action in (3.25) becomes

$$
\begin{aligned}
& -\int d t d^{p-1} y d w\left\{\frac{1}{4} \widetilde{G}_{\bar{\mu} \bar{\nu}}^{(n)} \widetilde{G}_{\bar{\mu} \bar{\nu}}^{(n)}+\frac{1}{2} n\left(C_{\bar{\mu}}^{(n)}-\partial_{\bar{\mu}} \varphi^{(n)}\right)^{2}\right. \\
& \left.\quad+\frac{1}{2} C_{\bar{\mu}}^{(n)}\left(-\partial_{w}^{2}+\frac{1}{4} w^{2}-\frac{1}{2}\right) C_{\bar{\mu}}^{(n)}+\frac{1}{2} n \varphi^{(n)}\left(-\partial_{w}^{2}+\frac{1}{4} w^{2}-\frac{1}{2}\right) \varphi^{(n)}\right\},
\end{aligned}
$$

with the constraint

$$
\partial_{w}\left(\partial_{\bar{\mu}} B_{\bar{\mu}}^{(n)}-n \phi^{(n)}\right)=0 .
$$

This then is a nontrivial constraint on all modes that are not in the ground state of the $w$ coordinate harmonic oscillator. For the zero modes, as before, the solution for $C^{(n)}$ and $\varphi^{(n)}$ are such that the corresponding $B^{(n)}$ and $\phi^{(n)}$ fields are $w$ independent.

There is still a residual gauge invariance that acts on the lowest mode of the $C_{\bar{\mu}}^{(n)}$ fields, hence the lowest mass modes are massive vector fields on the $p-1$ brane. The gauge invariance does not extend to the higher modes. However, using the constraint in (3.27) and the equations of motion derived from (3.26), one can easily show that the 
higher modes satisfy the equations

$$
\begin{aligned}
-\partial^{\bar{\mu}} C_{\bar{\mu}}^{(n, k)}+n \varphi^{(n, k)} & =0 \\
-\partial_{\bar{\mu}} \partial^{\bar{\mu}} C_{\bar{\nu}}^{(n, k)}+(n+k) C_{\bar{\nu}}^{(n, k)} & =0 \\
-\partial_{\bar{\mu}} \partial^{\bar{\mu}} \varphi^{(n, k)}+(n+k) \varphi^{(n, k)} & =0
\end{aligned}
$$

where $k$ is the mass squared arising from the wavefunctions along the $y$ direction. Finally, we can define a new field $\widehat{C}_{\bar{\mu}}^{(n, k)}$ that is given by

$$
\widehat{C}_{\bar{\mu}}^{(n, k)}=C_{\bar{\mu}}^{(n, k)}-\frac{n}{n+k} \partial_{\bar{\mu}} \varphi^{(n, k)} .
$$

Hence, the first and last equations in $(\overline{3.28})$ give $\partial_{\bar{\mu}} \widehat{C}_{\bar{\mu}}^{(n, k)}=0$. Thus, the higher modes on the $p-1$ brane have massive vectors $\widehat{C}_{\bar{\mu}}^{(n, k)}$, and massive scalars $\varphi^{(n, k)}$.

\section{Comparison to the bosonic D-brane spectrum}

In a field theory with just a tachyon, the fluctuation modes on the unstable lump are nondegenerate. We know of course that open string modes on D branes are highly degenerate. The original tachyon model, with the nice feature of having a discrete spectrum with the correct spacing between levels, could not fail to miss most of the states that one would expect from string theory. By adding in a gauge field, however, there are more fluctuation modes, enough in fact to start making reasonable conjectures as to how they match with the open strings on a D-brane.

The purpose of this section is to do just that and compare the results for the tachyon and gauge field fluctuations of the previous section to results for the D brane spectrum in bosonic string theory. We find that our results fit nicely with a subclass of oscillator states for the D brane fluctuations of the bosonic string. This attests to the power of these models and strongly suggests that the original model can be made into a complete string model by adding the rest of the fields.

In comparing the string theory construction of the spectrum on a D25-brane and on a D24-brane, we know that (apart from momentum modes which differ) the number of physical degrees of freedom are the same and just rearrange themselves into different representations of the Lorentz group. For example the first three levels of the D25 contain the tachyon, a massless vector, and a massive symmetric rank two tensor respectively. On the D24 brane, these turn into a tachyon, a massless vector and a massless scalar, and at the next level, a massive symmetric rank two tensor, a massive vector and a massive 
scalar. In our discussion, where the D24 appears as a solution of the D25 field theory, we have seen that the D25 tachyon localizes to a D24 tachyon, plus a tower of higher mass squared D24 scalars. The D25 gauge field localizes to a D24 massless gauge field and a tower of D24 massive gauge fields. Although we have not made a localization analysis for the massive symmetric rank two tensor on the D25, its Stueckelberg formulation 29] suggests strongly that it localizes on the D24 to an equal mass symmetric rank two tensor (plus additional states at higher masses). We therefore see a nice pattern appearing, the fluctuations of the tachyon field on the D24 provide the scalars needed both at the massless and higher mass levels. In addition, the fluctuations of the gauge field on the D24 provides the massive gauge field needed at the next level. In summary, modes "lost" by localization are obtained as fluctuation modes of lower mass fields.

In order to provide further insight into this subject we attempt to identify the particular oscillator states that correspond to the various localized fields. To this end consider then the D25 brane of bosonic string theory. The tachyon state is the string ground state $|\Omega\rangle=c_{1}|0\rangle$ and the gauge states are $\alpha_{-1}^{\mu}|\Omega\rangle$. Notice that under the twist operation $\sigma \rightarrow \pi-\sigma$, the oscillators transform as

$$
\alpha_{n}^{\mu} \rightarrow(-1)^{n} \alpha_{n}^{\mu}
$$

The tachyon state twist even and hence the gauge field states are twist odd. In the string field theory the fluctuation modes of a field should have the same twist as the field itself. If we now consider lower dimensional D branes, then the oscillators with components transverse to the brane have their twist flipped, so that

$$
\widetilde{\alpha}_{n}^{I} \rightarrow-(-1)^{n} \widetilde{\alpha}_{n}^{I},
$$

where $\widetilde{\alpha}_{n}^{I}$ refers to a transverse oscillator. Hence for the D24 brane, the states

$$
\left(\widetilde{\alpha}_{-1}^{25}\right)^{k}|\Omega\rangle
$$

are all twist even states. It is these states that we propose should be identified with the fluctuations of the tachyon. For higher codimension branes, the tachyon fluctuations are identified with the states built with $\widetilde{\alpha}_{-1}^{I}$ oscillators acting on $|\Omega\rangle$. Indeed, with $c$ transverse dimensions, this matches correctly with the spectrum of the $c$-dimensional simple harmonic oscillator, which is the spectrum of the tachyon fluctuations around the codimension $c$ lump solution (see [9] section 5). 
Next consider matching the gauge fields on the D24 brane. We suggest the identification

$$
\alpha_{-1}^{\hat{\mu}}|\Omega\rangle \Longleftrightarrow B_{\hat{\mu}}^{(0)}, \quad \hat{\mu}=0,1, \cdots 24,
$$

with the massless gauge field $B_{\hat{\mu}}^{(0)}$ of the previous section. For the massive gauge fields we propose

$$
\left\{\alpha_{-1}^{\mu}\left(\widetilde{\alpha}_{-1}^{25}\right)^{n}|\Omega\rangle, \widetilde{\alpha}_{-2}^{25}\left(\widetilde{\alpha}_{-1}^{25}\right)^{n-1}|\Omega\rangle\right\} \Longleftrightarrow\left\{B_{\hat{\mu}}^{(n)}, \phi^{(n)}\right\} \quad n=1,2, \cdots
$$

where the identification uses the Stueckelberg formulation of (3.25). Note that all the above states are twist odd, as they ought to be since they arise from the twist odd gauge field.

Since we obtained in the previous section the localization of a massive gauge field (giving an equal mass gauge field and at each higher level a gauge field plus a scalar) we can examine the gauge fields on the D23, as obtained by localizing all the gauge fields on the D24. Again the massless vector on the D23 is identified $\alpha_{-1}^{\bar{\mu}}|\Omega\rangle$, with $\bar{\mu}=0,1, \cdots 23$. This is the lowest mode arising from the localization of the D24 field $B_{\hat{\mu}}^{(0)}$. The higher massive gauge field modes arising from the D24 field $B_{\hat{\mu}}^{(0)}$ are identified as

$$
\left\{\alpha_{-1}^{\bar{\mu}}\left(\widetilde{\alpha}_{-1}^{24}\right)^{k}|\Omega\rangle, \widetilde{\alpha}_{-2}^{24}\left(\widetilde{\alpha}_{-1}^{24}\right)^{k-1}|\Omega\rangle\right\} \Longleftrightarrow\left\{C_{\bar{\mu}}^{(0, k)}, \varphi^{(0, k)}\right\}, \quad k=1,2, \cdots,
$$

where comparing with (3.26) we see that in this case the $\varphi^{(0, k)}$ are Stueckelberg fields (the last term in (3.26) vanishes and the constraint in (3.27) is empty). The remaining gauge field states can be associated with states arising from the localization of the massive D24 gauge fields $B_{\hat{\mu}}^{(n)}$, with $n=1,2, \cdots$. They are:

$$
\left\{\left(\alpha_{-1}^{\bar{\mu}}\left(\widetilde{\alpha}_{-1}^{25}\right)^{n}\left(\widetilde{\alpha}_{-1}^{24}\right)^{k}, \widetilde{\alpha}_{-2}^{25}\left(\widetilde{\alpha}_{-1}^{25}\right)^{n-1}\left(\widetilde{\alpha}_{-1}^{24}\right)^{k}\right), \widetilde{\alpha}_{-2}^{24}\left(\widetilde{\alpha}_{-1}^{25}\right)^{k}\left(\widetilde{\alpha}_{-1}^{24}\right)^{n-1}\right\} \Leftrightarrow\left\{C_{\bar{\mu}}^{(n, k)}, \varphi^{(n, k)}\right\},
$$

where $k=0,1,2, \cdots$. The fields $\left(C_{\bar{\mu}}^{(n, k)}, \varphi^{(n, k)}\right)$ in $(3.26)$ define for $k>0$ a massive gauge field formulated without its Stueckelberg partner, and a massive scalar. We see in the above equation that, as usual, oscillator states in string theory provide for the Stueckelberg partners, in addition to the scalars. For $k=0$, there is no extra scalar, and indeed we see that in that case the last state in the above left hand side had already been listed in (4.6) and therefore is not available. This concludes our discussion of the identification of localized gauge states with D-brane open string states. 


\section{$5 \quad$ Fermion fields in the superstring tachyon model}

For the unstable D9 brane of Type IIA string theory, there are 16 massless fermionic degrees of freedom described by modes one 10 dimensional Majorana fermion. For the BPS D8 brane there are 8 massless fermionic degrees of freedom described by one 9 dimensional Majorana fermion that pairs up with a massless scalar and gauge field to form a supermultiplet [24]. We would like to see how this happens in general and in the string field theory models in particular.

We thus consider the following action for a fermion field

$$
S=-\int d t d^{9} x\left(\mathcal{K}^{\prime}(T)\right)^{2}\left[\frac{i}{2} \bar{\psi} \Gamma^{\mu} \stackrel{\leftrightarrow}{\partial}_{\mu} \psi+W(T) \bar{\psi} \psi\right]
$$

where

$$
W(T)=-\frac{\mathcal{K}^{\prime \prime}(T)}{\mathcal{K}^{\prime}(T)}
$$

and compute the fermion spectrum about the kink solution $T=x$. The analysis closely follows classic work on the study of fermions on a soliton background [30. To this end we define the field $\chi=\mathcal{K}^{\prime}(T) \psi$, in which case the fermion action in the soliton background reduces to

$$
S=-\int d t d^{8} y d x\left[i \bar{\chi} \Gamma^{\mu} \partial_{\mu} \chi+W(x) \bar{\chi} \chi\right] .
$$

Hence, the prefactor in (5.1) plays no role in determining the fluctuation spectrum, leaving its precise form ambiguous in this analysis. We adopted the specific form in (5.1) in order to have an action with the same structural form as the tachyon and gauge field action.

We then choose a basis for the $\Gamma$ matrices

$$
\Gamma^{x}=\left(\begin{array}{cc}
0 & i I \\
i I & 0
\end{array}\right) \quad \Gamma^{\mu}=\left(\begin{array}{cc}
\gamma^{\mu} & 0 \\
0 & -\gamma^{\mu}
\end{array}\right) \quad \mu=0 \ldots 8
$$

where $\gamma^{\mu}$ refers to the 9 dimensional $\Gamma$ matrices. The fermion spectrum is determined by finding the eigenvalues of the linear equation

$$
\left(\begin{array}{cc}
W(x) & -\frac{d}{d x} \\
-\frac{d}{d x} & W(x)
\end{array}\right)\left(\begin{array}{l}
\chi_{1} \\
\chi_{2}
\end{array}\right)=m\left(\begin{array}{c}
\chi_{1} \\
-\chi_{2}
\end{array}\right)
$$

where the sign on the rhs of (5.5) arises because of the form of $\Gamma^{\mu}$ in (5.4). Hence, we find that the linear combinations $\chi_{ \pm}=\chi_{1} \pm \chi_{2}$ satisfy the equations

$$
\left[-\frac{d^{2}}{d x^{2}}+W^{2}(x) \pm W^{\prime}(x)-m^{2}\right] \chi_{ \pm}=0 .
$$


Thus, we find that $\chi_{-}$and $\chi_{+}$satisfy the two different Schroedinger equations

$$
\begin{array}{r}
\left(-\frac{d^{2}}{d x^{2}}+\frac{\mathcal{K}^{\prime \prime \prime}(x)}{\mathcal{K}^{\prime}(x)}-m^{2}\right) \chi_{-}=0, \\
\left(-\frac{d^{2}}{d x^{2}}+2\left(\frac{\mathcal{K}^{\prime \prime}(x)}{\mathcal{K}^{\prime}(x)}\right)^{2}-\frac{\mathcal{K}^{\prime \prime \prime}(x)}{\mathcal{K}^{\prime}(x)}-m^{2}\right) \chi_{+}=0 .
\end{array}
$$

The Schroedinger equation for the $\chi_{-}$modes is the same as for the tachyon fluctuations (see eq. (2.11)). Hence, in this case the lowest mode is massless. The Schroedinger equation for the $\chi_{+}$modes is different from the tachyon fluctuations, the potential differing by $2 W^{\prime}(x)$. In general, $W^{\prime}(x)$ is a positive definite function, so these modes are all massive. Therefore, since only half the fermion degrees of freedom have massless modes, we find that there are 8 massless fermion modes on the kink background.

Let us now specialize to the finite $\ell$ models. Using eq. (2.7) we have that

$$
W_{\ell}(T)=\ell \tanh (T)
$$

Therefore, the potential term in the second line of (5.7) is

$$
2\left(\frac{\mathcal{K}^{\prime \prime}(x)}{\mathcal{K}^{\prime}(x)}\right)^{2}-\frac{\mathcal{K}^{\prime \prime \prime}(x)}{\mathcal{K}^{\prime}(x)}=\ell^{2}-\ell(\ell-1) \operatorname{sech}(x)=2 \ell-1+U_{\ell-1}(x),
$$

in other words the mass spectrum for these fermion modes is derived from the $\ell-1$ model, shifted by a positive integer.

Next consider the IIA model, where $\mathcal{K}^{\prime}(T)$ is given by 2.27$)$ and so $W(T)=T / 2$. In this case the action in (5.1) becomes

$$
S=-\mathcal{T} \int d t d^{9} x \exp \left(-T^{2} / 2\right)\left[\frac{i}{2} \bar{\psi} \Gamma^{\mu} \stackrel{\leftrightarrow}{\partial}_{\mu} \psi+\frac{1}{2} T \bar{\psi} \psi\right]
$$

The Yukawa coupling in (5.10) can be justified by considering a three string amplitude. Moreover, a term of this sort lifts the fermion mass to infinity as the tachyon rolls to the closed string vacua at $x= \pm \infty$. The mode equations now reduce to

$$
\left[-\frac{d^{2}}{d x^{2}}+\frac{1}{4} x^{2} \pm \frac{1}{2}-m^{2}\right] \chi_{ \pm}=0 .
$$

Therefore, the $\chi_{+}$modes have their levels shifted by one unit, with the lowest mode starting at $m^{2}=1$.

We have shown that the action in (5.1) leads to 8 massless fermion modes, hence the full model has bose-fermi degeneracy at the massless level. However, the massive 
states do not have this degeneracy. In order to achieve this, as well as a full space-time supersymmetry, one will need to include an infinite number of fields in the string field theory action.

\section{Higher derivative actions in the superstring tachyon model}

The tachyon action that models the unstable D9 brane of IIA in (2.26) is of course only an approximation to the true string field theory. Higher derivative terms are indeed present in the complete action. The authors of [13] have argued that the action for a kink solution of the form $T=u x$ has the form

$$
S=-\frac{\mathcal{T} \sqrt{\pi q} 4^{q}(\Gamma(q))^{2}}{\sqrt{2} \Gamma(2 q)}, \quad T=u x, \quad q \equiv u^{2} .
$$

The action of the kink is minimized if $q \rightarrow \infty$, thus shrinking the width of the kink to zero size. The action in (6.1) clearly has higher derivative terms, although it is consistent with an action that only has a single derivative acting on each $T$ field.

\subsection{Higher derivative actions}

Let us generalize the action in (2.26) to be of the form?

$$
S=-\mathcal{T} \int d t d^{p+1} x e^{-T^{2} / 2} f\left(\partial_{\mu} T \partial^{\mu} T\right)
$$

We will assume for normalization purposes, and in order to have a nonvanishing term with two derivatives leading to a tachyon, that

$$
f(0)=1, \quad f^{\prime}(0)>0 .
$$

Indeed, with these conditions one readily finds that there is a tachyon around the $T=0$ vacuum, with mass squared:

$$
M_{T}^{2}=-\frac{1}{2 f^{\prime}(0)}
$$

\footnotetext{
${ }^{6}$ We have set $\alpha^{\prime}=1$.

${ }^{7}$ The solvability of tachyonic actions with higher derivatives was also noticed by Ashoke Sen [31].
} 
One can easily see that $T=u x$ is a kink solution provided that

$$
2 q f^{\prime}(q)=f(q), \quad q=u^{2}
$$

This equation should be viewed as a constraint on the choice for a function $f$ and on the value $u$ used in the kink solution. Finally, we can also compute for this action the ratio of brane tensions. By the definition of the action (6.2), we have $\mathcal{T}_{p}=\mathcal{T}$, and splitting $d^{p+1} x=d^{p} y d x$ we find

$$
\mathcal{T}_{p-1}=\mathcal{T} \int d x e^{-q x^{2} / 2} f(q)=\mathcal{T} \sqrt{\frac{2 \pi}{q}} f(q)
$$

This leads to

$$
\frac{\sqrt{2}}{2 \pi} \frac{\mathcal{T}_{p-1}}{\mathcal{T}_{p}}=\frac{1}{\sqrt{\pi}} \frac{f(q)}{\sqrt{q}},
$$

which in string theory must take the value of unity.

As a small check, we verify that this more general setup reproduces the results obtained before. For example, the action (2.26) corresponds to $f(q)=1+q$, which solves (6.5) for $q=1 \rightarrow u= \pm 1$. In this case $M_{T}^{2}=-1 / 2$ follows from (6.4), and the ratio in (6.7) takes the value $2 / \sqrt{\pi}$, all this in agreement with [10].

We can now investigate the small fluctuations problem about the solution of the general action. To this end, let $T=u x+\widetilde{T}$. Expanding the action in (6.2) to second order in $\widetilde{T}$, using (6.5), redefining the fluctuation field as $\widetilde{T}=\widehat{T} e^{u^{2} x^{2} / 4}$, and integrating by parts, one finds

$$
\begin{aligned}
S & =-\mathcal{T} \int d t d^{p} y d x\left(e^{-q x^{2} / 2} f(q)+\frac{4 f(q)}{q}\left\{\frac{1}{2} \partial_{\mu} \widehat{T} \partial^{\mu} \widehat{T}\right.\right. \\
& \left.\left.+\frac{1}{2}\left(1+4 q^{2} \frac{f^{\prime \prime}(q)}{f(q)}\right)\left[\left(\partial_{x} \widehat{T}\right)^{2}+\frac{1}{4} x^{2} q^{2} \widehat{T}^{2}-\frac{1}{2} q \widehat{T}^{2}\right]\right\}\right) .
\end{aligned}
$$

Thus the spectrum of the small fluctuations about the kink are given by the eigenvalues of the one dimensional harmonic oscillator, as can be seen from the second line in the above formula. The lowest mode is massless and the mass squared spacing is given by

$$
\Delta m^{2}=q\left(1+4 q^{2} \frac{f^{\prime \prime}(q)}{f(q)}\right) .
$$

At this point the we can summarize the constraints that can be imposed on the model presented in eqn. 6.2 ): 
- Getting the string theory tachyon mass (see (6.4)),

- Getting the string theory ratio of brane tensions (see (6.7)),

- Getting the string theory mass squared spacing (see (6.9)).

In fact, these conditions are not enough to fix uniquely the function $f$. On the other hand, it seems clear that the class of models we are considering do not lead to the the complete boundary string field theory action for the tachyon, which is expected to have more general patters of higher derivative terms. In order to see this we demand that, as in B-SFT, that the kink solution arises in the limit $u \rightarrow \infty$. Examining (6.5) we see that $f(q) \sim \sqrt{q}$ as $q \rightarrow \infty$. Furthermore, we see from (6.8) that this asymptotic behavior for $f(q)$ is necessary to insure a finite mass spectrum.

We now consider two examples for the function $f(q)$. The first is that derived from the action in (6.1). In this case $f(q)$ is given by

$$
f(q)=\frac{q 4^{q}(\Gamma(q))^{2}}{2 \Gamma(2 q)}=\sqrt{\pi q}+\frac{1}{8} \frac{\sqrt{\pi}}{\sqrt{q}}+\mathrm{O}\left(q^{-3 / 2}\right) .
$$

This leads to a mass splitting that is half the expected value. The likely conclusion is that there are higher derivative terms for the fluctuations.

The second example is derived from the modified Born-Infeld action proposed in [27, 28]. The tachyon kinetic term is incorporated into the action to give the following:

$$
S=-\mathcal{T} \int d t d^{p+1} x V(T) \sqrt{-\operatorname{det}\left(\eta_{\mu \nu}+F_{\mu \nu}+2 \partial_{\mu} T \partial_{\nu} T\right)} .
$$

In order to match to the potential of B-SFT, we set

$$
V(T)=\exp \left(-T^{2} / 2\right)
$$

With the gauge field absent, the determinant satisfies the relation

$$
-\operatorname{det}\left(\eta_{\mu \nu}+2 \partial_{\mu} T \partial_{\nu} T\right)=1+2 \partial_{\mu} T \partial^{\mu} T .
$$

Therefore, we can use the above arguments to find the spectrum for the tachyon field fluctuations, with

$$
f(q)=(1+2 q)^{1 / 2} .
$$

\footnotetext{
${ }^{8}$ In the complete B-SFT the descent relations hold exactly [11, 13, 32].
} 
Strictly speaking, there is no $q$ that is a solution to eq. 6.5) for this $f(q)$. Instead, we can modify the function to

$$
f_{\epsilon}(q)=(1+2 q)^{\frac{1}{2}+\epsilon}
$$

and take the limit $\epsilon \rightarrow 0$. In this limit, the solution of (6.5) is $q=2 / \epsilon \rightarrow \infty$, and so the width of the brane is shrinking to zero size. Choosing the function in $(\sqrt{6.15})$ and taking the limit, we see that the open string tachyon mass is $m^{2}=-1 / 2$. We also see from (6.9) that the spacing between mass levels is twice the magnitude of the tachyon mass squared. However, the tension of the 8-brane as compared to that of the unstable 9-brane is $2 \sqrt{\pi}$ which is less than the actual value of $\sqrt{2} \pi$.

\subsection{Gauge fluctuations for the modified Born-Infeld action}

In this section we compute the spectrum for the gauge fluctuations on a kink background for the action in (6.11). Setting $T$ to the kink solution $T=u x$ and expanding the determinant in (6.11) to second order in $F_{\mu \nu}$, we have

$$
-\operatorname{det}\left(\eta_{\mu \nu}+F_{\mu \nu}+2 \partial_{\mu} T \partial_{\nu} T\right)=\left(1+2 u^{2}\right)\left(1-\frac{1}{2} F_{\mu \nu} N^{\nu \lambda} F_{\lambda \delta} N^{\delta \mu}\right)+\ldots,
$$

where

$$
N^{\mu \nu}=\eta_{\mu \nu}-\frac{2 u^{2}}{1+2 u^{2}} \delta_{\mu x} \delta_{\nu x}
$$

Hence, up to second order in fluctuations we find

$$
-\operatorname{det}\left(\eta_{\mu \nu}+F_{\mu \nu}+2 \partial_{\mu} T \partial_{\nu} T\right)=1+\frac{1}{2}\left(1+2 u^{2}\right) F_{\hat{\mu} \hat{\nu}} F_{\hat{\mu} \hat{\nu}}+F_{\hat{\mu} x} F_{\hat{\mu} x} .
$$

To find the gauge fluctuations about the kink background, we plug (6.18) into (6.11) and expand to second order, giving

$$
-\mathcal{T} \int d t d^{p} y d x \exp \left(-u^{2} x^{2} / 2\right)\left(1+2 u^{2}\right)^{1 / 2}\left(\frac{1}{4} F_{\hat{\mu} \hat{\nu}} F_{\hat{\mu} \hat{\nu}}+\frac{1}{2\left(1+2 u^{2}\right)} F_{\hat{\mu} x} F_{\hat{\mu} x}\right) .
$$

Following the arguments in section 3 , we find that the spectrum has equally spaced levels with spacing

$$
\Delta m^{2}=\frac{u^{2}}{1+2 u^{2}}=\frac{q}{1+2 q} .
$$

Therefore, in the limit $q \rightarrow \infty$, the spacing of the levels is half the expected value.

While not the desired value, we note that the spacing we found is finite. If the BornInfeld action was of the usual type, without the tachyon kinetic term, then for a kink of the form $T=u x$, the spacing would have been $u^{2}=q$. If $q \rightarrow \infty$, as is expected in B-SFT, this would push the spacing to infinity. That it stays finite seems to provide some support for the form of the action in (6.11). 


\subsection{Higher codimension branes}

We can also consider higher codimension branes. In the models for the bosonic string tachyon such solutions existed exploiting the fact that the tachyon potential is unbounded below. Since the tachyon potential for the superstring is positive definite we need a different way to avoid Derrick's no-go result for higher codimension branes. This would seem to require several tachyon fields and gauge fields. However, Derrick's theorem assumes a standard kinetic operator for the scalar fields. If there are higher derivative terms then higher codimension objects appear to be possible when we have several tachyon fields, even without exciting the gauge fields.

In order to construct these higher codimension branes, we thus start with more than one D9 brane. If we have $N$ such branes, then the tachyons transform in the adjoint of $U(N)$. For a codimension $d$ brane, the tachyon configuration has the form [1], 33, 34, 13]

$$
T=\Gamma_{m} u x^{m},
$$

where the index $m$ refers to the transverse directions and the $\Gamma_{m}$ are the $d$ dimensional Dirac matrices. The tachyon configuration in (6.21) is a solution to the equations of motion for the action

$$
S=-\mathcal{T} \int d t d^{p+1} x \operatorname{Tr}\left[e^{-T^{2} / 2} f\left(\partial_{\mu} T \partial^{\mu} T\right)\right] .
$$

with $f$ satisfying

$$
2 \frac{q}{d} f^{\prime}(q)=f(q),
$$

where $q=d u^{2}$. If we use the two derivative action in (2.26), then we see that there is no solution for $d>2$ and that in the limit $d \rightarrow 2, q$ is pushed out to infinity.

However, including higher derivative terms leads to solutions with finite values of $q$. For instance, suppose that we choose $f(q)$ to be

$$
f(q)=(1+q / d)^{d} .
$$

This choice for $f(q)$ leads to a tachyon mass with $m^{2}=-1 / 2$. It has a solution at $q=d$, which corresponds to $u=1$. Computing the tension of the codimension $d$ brane, we find that

$$
T_{9-d}=2^{d / 2} 2^{d}(2 \pi)^{d / 2} \mathcal{T} .
$$

The total tension of the 9 branes is $2^{d / 2} \mathcal{T}$, which takes into account the multiple unstable branes or brane-antibrane pairs, depending on whether $d$ is odd or even. Hence the ratio 
of tensions to a single IIB 9 brane is

$$
\frac{T_{9-d}}{T_{9}}=2^{d}(4 \pi)^{d / 2}=\left(\frac{4}{\pi}\right)^{d / 2}(2 \pi)^{d} .
$$

Hence, this choice for $f(q)$ leads to constant descent relations and can basically be thought of as the extension to the two derivative truncation for lower dimensional branes.

\section{Conclusions}

We have extended the previously constructed superstring tachyon effective theory [10] to include gauge fields and fermions. The complete model, assembled from (2.14) and (5.1) reads

$$
S=-\int d t d^{p+1} x\left(\mathcal{K}^{\prime}(T)\right)^{2}\left[(\partial T)^{2}+1+\frac{1}{4} F^{\mu \nu} F_{\mu \nu}+\frac{i}{2} \bar{\psi} \Gamma^{\mu} \stackrel{\leftrightarrow}{\partial}_{\mu} \psi+W(T) \bar{\psi} \psi\right],
$$

where $\mathcal{K}^{\prime}(T)=\sqrt{\mathcal{T}} \exp \left(-T^{2} / 4\right), W(T)=T / 2$, and we work with $p=8$ for the unstable D9 of IIA. This action gives the correct tachyon mass, and correct mass spectrum for the tachyon, gauge field and fermion field fluctuations around the kink solution representing a codimension one brane. On the other hand, the descent relation for the tension is not exactly that of string theory. As we have explained, the above interactions for gauge fields and fermion fields result in solvable spectra around brane configurations, but any action for gauge fields or fermions that is equivalent to this action, up to quadratic order in those fields, will have the same solvability properties and exactly the same spectrum. This includes, in particular, Born-Infeld actions of the form

$$
S=-\int d t d^{p+1} x\left(\mathcal{K}^{\prime}(T)\right)^{2} \sqrt{-\operatorname{det}\left(\eta_{\mu \nu}+F_{\mu \nu}\right)}+\cdots
$$

where the dots denote tachyon and fermion kinetic terms. In fact, our analysis of actions with higher derivatives in section 6 shows that solvability is still possible if, following [27, 28], we include the tachyon kinetic term inside the square root giving

$$
S=-\int d t d^{p+1} x\left(\mathcal{K}^{\prime}(T)\right)^{2} \sqrt{-\operatorname{det}\left(\eta_{\mu \nu}+F_{\mu \nu}+2 \partial_{\mu} T \partial_{\nu} T\right)}+\text { fermions }
$$

Details of the solutions and spectra, however, differ from that of $(7.27)$, since the tachyon background is changed (it is $T=u x$, with $u \rightarrow \infty$ ). In particular, the level spacing for the gauge field fluctuations is half the expected one. 
For the bosonic string theory tachyon model the two alternative forms of the action are based on equations (3.13) and (3.18)

$$
S=-\int d t d^{p+1} x\left(\mathcal{L}^{\prime}(T)\right)^{2}\left(\frac{1}{2}(\partial T)^{2}+2 T+\left\{\begin{array}{r}
2 T \\
1
\end{array}\right\} \frac{1}{4} F_{\mu \nu} F^{\mu \nu}\right),
$$

with $\mathcal{L}^{\prime}(T)=\frac{1}{2} \sqrt{\mathcal{T} e} \exp (-T / 4)$. The first form is Born-Infeld compatible and has finite $\ell$ solvable counterparts. The spectrum of gauge fluctuations, however, has a mass spacing which is twice the expected one. The second form is not Born-Infeld compatible and does not have finite $\ell$ solvable counterparts. On the other hand the mass spacing is the expected one. Note that neither form is compatible with tachyon kinetic terms inside the Born-Infeld square root, since the prefactor in the tachyon kinetic term does not coincide with the tachyon potential. It is not completely clear how to decide between these two possibilities, but it seems reasonable to choose the form with correct mass spacing. As reviewed in the introduction, both forms have appeared in B-SFT studies.

In section (6.2) we saw that one could construct stable solutions on the worldvolume of coincident branes representing branes with codimension greater than one without turning on gauge fields. This was possible because the tachyon field theory model used had higher derivative terms for the tachyon, and is why such solutions could be found also in B-SFT [13]. The existence of such solutions, however, is somewhat puzzling as earlier discussions of configurations on the world volume of coincident D-branes leading to a lower dimensional stable branes appear to require non-trivial gauge field backgrounds [1, 33, 34.

The surprising and pleasant features of the original tachyon models [9, 10] were that simple field theory interactions exactly described a situation where the tachyon could dissappear completely in the stable vacuum and where nontrivial configurations representing branes existed with a fluctuation spectrum of stringy type. In retrospect, we see that such properties essentially guaranteed agreement between these models and the two-derivative truncations of B-SFT formulations of tachyon dynamics. We have seen in this paper that these pleasant features nicely extend to models including the interactions of the tachyon with gauge and fermion fields.

Acknowledgments: J.A.M. would like to thank the CTP at MIT and the theory group at Harvard for hospitality during the course of this work. We are grateful to Jeffrey Goldstone for discussions on fermion localization on kinks. We also wish to thank Igor 
Klebanov and Leonardo Rastelli for their comments on gauge invariance in B-SFT. Finally, we would like to thank Ashoke Sen for many useful remarks and discussions. This work was supported in part by DOE contract \#DE-FC02-94ER40818.

\section{References}

[1] A. Sen, "Descent relations among bosonic D-branes," Int. J. Mod. Phys. A14, 4061 (1999) hep-th/9902105; "Stable non-BPS bound states of BPS D-branes," JHEP 9808, 010 (1998) hep-th/9805019;; "Tachyon condensation on the brane antibrane system," JHEP 9808, 012 (1998) hep-th/9805170; "SO(32) spinors of type I and other solitons on brane-antibrane pair," JHEP 9809, 023 (1998) hep-th/9808141.

[2] E. Witten, "Noncommutative Geometry And String Field Theory," Nucl. Phys. B268, 253 (1986).

[3] N. Berkovits, "Super-Poincare Invariant Superstring Field Theory," Nucl. Phys. B450 (1995) 90, [hep-th/9503099].

[4] V. A. Kostelecky and S. Samuel, "On A Nonperturbative Vacuum For The Open Bosonic String," Nucl. Phys. B336, 263 (1990);

A. Sen and B. Zwiebach, "Tachyon condensation in string field theory," JHEP 0003, 002 (2000) hep-th/9912249.

W. Taylor, "D-brane effective field theory from string field theory", hep-th/0001201.

N. Moeller and W. Taylor, "Level truncation and the tachyon in open bosonic string field theory", hep-th/0002237.

J.A. Harvey and P. Kraus, "D-Branes as unstable lumps in bosonic open string field theory", hep-th/0002117.

R. de Mello Koch, A. Jevicki, M. Mihailescu and R. Tatar, "Lumps and p-branes in open string field theory", hep-th/0003031.

N. Moeller, A. Sen and B. Zwiebach, "D-branes as tachyon lumps in string field theory," hep-th/0005036.

R. de Mello Koch and J. P. Rodrigues, "Lumps in level truncated open string field theory," hep-th/0008053.

N. Moeller, "Codimension two lump solutions in string field theory and tachyonic theories," hep-th/0008101. 
A. Sen and B. Zwiebach, "Large marginal deformations in string field theory," hepth/0007153.

W. Taylor, "Mass generation from tachyon condensation for vector fields on Dbranes," hep-th/0008033.

[5] N. Berkovits, "The Tachyon Potential in Open Neveu-Schwarz String Field Theory," hep-th/0001084;

N. Berkovits, A. Sen and B. Zwiebach, "Tachyon condensation in superstring field theory", hep-th/0002211;

P. De Smet and J. Raeymaekers, "Level four approximation to the tachyon potential in superstring field theory", hep-th/0003220;

A. Iqbal and A. Naqvi, "Tachyon condensation on a non-BPS D-brane," hepth/0004015; "On marginal deformations in superstring field theory," hep-th/0008127.

[6] A. Sen, "Universality of the tachyon potential," JHEP 9912, 027 (1999) hepth/9911116;

L. Rastelli and B. Zwiebach, "Tachyon potentials, star products and universality," hep-th/0006240.

V. A. Kostelecky and R. Potting, "Analytical construction of a nonperturbative vacuum for the open bosonic string," hep-th/0008252.

H. Hata and S. Shinohara, "BRST invariance of the non-perturbative vacuum in bosonic open string field theory," hep-th/0009105.

B. Zwiebach, "Trimming the tachyon string field with SU(1,1)," hep-th/0010190.

[7] E. Witten, "On background independent open string field theory", Phys. Rev. D46, 5467 (1992) hep-th/9208027;

E. Witten, "Some computations in background independent off-shell string theory", Phys. Rev. D47, 3405 (1993) [hep-th/9210065];

K. Li and E. Witten, "Role of short distance behavior in off-shell open string field theory", Phys. Rev. D48, 853 (1993) hep-th/9303067.

[8] S.L. Shatashvili, "Comment on the background independent open string theory", Phys. Lett. B311, 83 (1993) hep-th/9303143; "On the problems with background independence in string theory", hep-th/9311177.

[9] J.A. Minahan and B. Zwiebach, "Field theory models for tachyon and gauge field string dynamics", JHEP 0009, 029 (2000) hep-th/0008231. 
[10] J. A. Minahan and B. Zwiebach, "Effective tachyon dynamics in superstring theory," hep-th/0009246.

[11] A.A. Gerasimov and S.L. Shatashvili, "On exact tachyon potential in open string field theory", hep-th/0009103.

[12] D. Kutasov, M. Marino and G. Moore, "Some exact results on tachyon condensation in string field theory", hep-th/0009148.

[13] D. Kutasov, M. Marino and G. Moore, "Remarks on tachyon condensation in superstring field theory," hep-th/0010108.

[14] D. Ghoshal and A. Sen, "Normalization of the background independent open string field theory action", hep-th/0009191.

[15] B. Zwiebach, "A solvable toy model for tachyon condensation in string field theory", CTP-MIT-3018, hep-th/0008227.

[16] F. Cooper, A. Khare and U. Sukhatme, "Supersymmetry and quantum mechanics," Phys. Rept. 251, 267 (1995) hep-th/9405029.

[17] J. Feinberg, "On kinks in the Gross-Neveu model," Phys. Rev. D51, 4503 (1995) hep-th/9408120;

J. Feinberg and A. Zee, "Dynamical Generation of Extended Objects in a 1+1 Dimensional Chiral Field Theory: Non-Perturbative Dirac Operator Resolvent Analysis," Phys. Rev. D56, 5050 (1997) cond-mat/9603173.

[18] J. A. Harvey and P. Kraus, "D-branes as unstable lumps in bosonic open string field theory," JHEP 0004, 012 (2000) hep-th/0002117.

[19] L. Cornalba, "Tachyon condensation in large magnetic fields with background independent string field theory," hep-th/0010021.

[20] K. Okuyama, "Noncommutative tachyon from background independent open string field theory," hep-th/0010028.

[21] O. Andreev, "Some computations of partition functions and tachyon potentials in background independent off-shell string theory," hep-th/0010218.

[22] A. A. Gerasimov and S. L. Shatashvili, "Stringy Higgs Mechanism and the Fate of Open Strings," hep-th/0011009. 
[23] A.A. Tseytlin, "Sigma model approach to string theory effective actions with tachyons," hep-th/0011033.

[24] A. Sen, "Supersymmetric world-volume action for non-BPS D-branes," JHEP 9910, 008 (1999) hep-th/9909062.

[25] P. Yi, "Membranes from five-branes and fundamental strings from Dp branes," Nucl. Phys. B550, 214 (1999) hep-th/9901159.

[26] O. Bergman, K. Hori and P. Yi, "Confinement on the brane," Nucl. Phys. B580, 289 (2000) hep-th/0002223.

[27] M. R. Garousi, "Tachyon couplings on non-BPS D-branes and Dirac-Born-Infeld action," Nucl. Phys. B584, 284 (2000) hep-th/0003122.

[28] E. A. Bergshoeff, M. de Roo, T. C. de Wit, E. Eyras and S. Panda, "T-duality and actions for non-BPS D-branes," JHEP 0005, 009 (2000) hep-th/0003221.

[29] W. Siegel and B. Zwiebach, "Gauge string fields," Nucl. Phys. B263, 105 (1986).

[30] R. Jackiw and C. Rebbi, "Solitons With Fermion Number 1/2," Phys. Rev. D13, 3398 (1976); C. Callias, "Index Theorems On Open Spaces," Commun. Math. Phys. 62, 213 (1978); J. Goldstone and F. Wilczek, "Fractional Quantum Numbers On Solitons," Phys. Rev. Lett. 47, 986 (1981).

[31] Ashoke Sen, private communication.

[32] S. Moriyama and S. Nakamura, "Descent relation of tachyon condensation from boundary string field theory," hep-th/0011002.

[33] E. Witten, "D-branes and K-theory," JHEP 9812, 019 (1998) hep-th/9810188.

[34] P. Horava, "Type IIA D-branes, K-theory, and matrix theory," Adv. Theor. Math. Phys. 2, 1373 (1999) hep-th/9812135. 